\title{
Open versus hybrid minimally invasive esophagectomy: Join the crowd, but do not throw away your abdominal retractors just yet
}

\author{
Wayne L. Hofstetter, MD
}

\footnotetext{
From the Department of Thoracic and Cardiovascular Surgery, University of Texas, MD Anderson Cancer Center, Houston, Tex.

No sources of funding are reported.

Received for publication March 30, 2019; revisions received May 18, 2019; accepted for publication May 22, 2019.

Address for reprints: Wayne L. Hofstetter, MD, Department of Thoracic and Cardiovascular Surgery, University of Texas, MD Anderson Cancer Center, 1515 Holcombe Blvd, Houston, TX 77005 (E-mail: whofstetter@ mdanderson.org)

J Thorac Cardiovasc Surg 2019;158:1475-8

0022-5223/\$36.00

Copyright (C) 2019 by The American Association for Thoracic Surgery

https://doi.org/10.1016/j.jtcvs.2019.05.090
}

Feature Editor's Note-Thoracic surgery is a specialty that embraces highly complex operative procedures while leading the universal field of minimally invasive surgery. Esophagectomy is arguably one of the most technically demanding gastrointestinal procedures, and its learning curve is not gentle. In the not too distant past, many would not have predicted that one-third of all esophagectomies performed by participants in the Society of Thoracic Surgeons database would be minimally invasive esophagectomies (MIEs). Esophagectomy is a procedure performed in at least 2 body cavities, often after neoadjuvant therapy, and whose many technical considerations directly impact the short-term, long-term, and likely cancer-specific recovery of our patients. Whereas many of these technical considerations are subtle, many are not. Esophagectomy is performed by a variety of approaches that encompass 2 versus 3 fields; cervical versus thoracic location of anastomosis; a variety of anastomotic techniques; and open, minimally invasive (thoracoscopic, laparoscopic, robotic), and hybrid techniques applied in a variety of ways. The study of outcomes (short- or long-term) after esophagectomy for any indication is complicated by the many permutations of these technical considerations, and rigor is limited outside the context of a clinical trial. Earlier this year, results from the MIRO randomized phase III trial were published in The New England Journal of Medicine and reported that a hybrid MIE procedure was associated with reduced postoperative complications compared with transthoracic open esophagectomy in patients with esophageal cancer. Whereas it has been my own personal bias that the sparing of a thoracotomy was the predominant benefit of MIE, the hybrid esophagectomy procedure in the

Bryan M. Burt, MD

hy $\cdot$ brid

/hī brid/

noun

AUTOMOTIVE automobile).

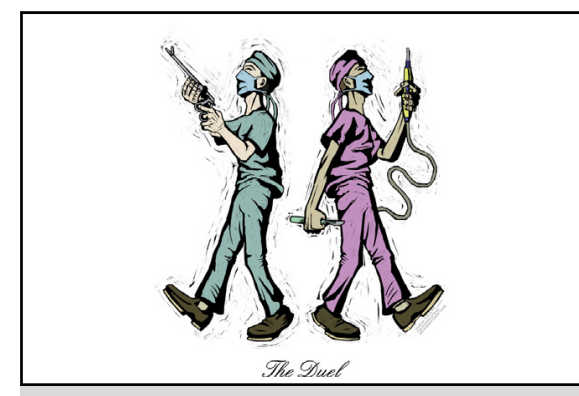

Open versus hybrid MIE. (c) 2019 The University of Texas M.D. Anderson Cancer Center.

Central Message

Randomized trials comparing open with minimally invasive esophagectomy (MIE) report equivalent to superior perioperative outcomes for MIE. A hybrid approach is a reasonable path to transition to MIE.

This Invited Expert Opinion provides a perspective on the following paper: $N$ Engl $J$ Med. 2019; 380:152-62. https://doi.org/10. 1056/NEJMoa1805101.

See Commentary on page 1479.

MIRO trial was defined by laparoscopy with thoracotomy. In the Feature Expert Opinion article that follows, the outcomes of this randomized trial are presented within the current framework of our understanding of the long-and short-term benefits of MIE and may challenge our preconceived notions about this procedure.

A car that runs on two different fuel sources (a mixture of an electric and gasoline engine

As in "I drive a hybrid." 
verb

\section{SURGERY}

The action of adopting modern surgical techniques while maintaining one foot in the realm of open surgery.

As in "Let's hybrid this one."

Minimally invasive approaches to esophagectomy for cancer have become a topic of abundant discussion as the techniques, tools, and learning curves have evolved. Somewhat of a novelty at the outset, minimally invasive esophagectomy (MIE) now pertains to a spectrum of surgical options practiced in hospitals worldwide. The adoption of these newer technologies seems to be related at least in part to the mounting evidence supporting equipoise and possible superiority relative to traditional open surgery that involves a laparotomy. In some recent randomized trials of hybrid MIE, researchers have focused on patient value ([outcomes - complications]/cost) while maintaining practices that are accessible to experienced esophageal surgeons.

A total MIE seemed to many groups to be the best way to avoid postoperative events caused by the trauma of opening 2 body cavities at the same surgery. Apart from favorable literature published by a few groups, however, others have shown the temerity to report a steep learning curve and less-than-optimal outcomes during the transition of open to MIE techniques. ${ }^{1-3}$ Issues related to the conduit interposition seemed to be the most challenging aspect of mastering a complete minimally invasive Ivor Lewis procedure. A hybrid esophagectomy (thoracotomy and laparoscopy), on the other hand, eliminates some of these challenges and is a rapid method of transitioning to MIE. It is interesting that past experience with the transhiatal operation singled out the thoracotomy as instigator of perioperative respiratory issues. In this era of enhanced recovery and neoadjuvant therapy, however, some groups have postulated counterintuitively that the combination of avoiding a laparotomy and using a small thoracotomy could actually be a key component of maintaining surgical quality while reducing operative and perioperative morbidity.

\section{RANDOMIZED CONTROLLED TRIALS Minimally Invasive Versus Open Oesophagectomy (TIME Trial)}

A seminal randomized controlled trial comparing total minimally invasive three-field versus open three-field esophagectomy, the "TIME" trial, was published in 2012. This trial consisted of a consortium of 5 European groups and included patients with locally advanced thoracic esophageal and upper esophagogastric junction tumors. ${ }^{4}$ Patients received neoadjuvant therapy (almost exclusively chemoradiation). Primary end points of in-hospital postoperative pulmonary infection significantly favored the hybrid relative to the open esophagectomy group (12\% vs $34 \%$ ). Secondary end points of length of stay, blood loss, and patient-recorded outcome measures also strongly favored the minimally invasive arm. Long-term overall survival was later reported as equivalent at 3 year follow-up. ${ }^{5}$ The additional question of whether patients in the open operation arm of the trial "catch up" after recovery from the initial decline of surgery was addressed with a 1-year assessment of quality of life survey. ${ }^{6}$ Results of these surveys indicated that, after a year of follow-up, the minimally invasive cohort reported superior quality of life indices. This outcome measure bears repetition; even after a year, the minimally invasive group reported better quality of life than did the open cohort.

The fact that this was a relatively small trial (just more than 100 patients randomized) and that the publication was released at a time when a growing body of literature on similar retrospective comparisons were reporting equipoise rather than superiority meant that there was room for interpretation and criticism of the TIME trial conclusions. I do not actually believe that there was doubt that the minimally invasive groups were performing better than the 3-field open group in this particular trial; the question was whether the open surgery, control group was performing to what would be considered an "industry standard." 7 Nothing makes a therapeutic arm look better than a poorly performing control arm. It was with this background that I, and certainly many others treating esophageal cancer, eagerly awaited the results of the MIRO trial, a slightly larger randomized control trial comparing hybrid MIE with open surgery. ${ }^{8}$ This trial would avoid the potential morbidity issues related to 3-field operation, instead focusing on the differences of laparotomy versus laparoscopy as the abdominal component of a 2-field operation.

\section{Hybrid Minimally Invasive Esophagectomy for Esophageal Cancer (MIRO Trial)}

In the MIRO trial, the patient population was limited to those with lower thoracic and type I esophagogastric junction tumors with either squamous or adenocarcinoma histology. Patients were predominantly treated with neoadjuvant therapy (chemotherapy or chemoradiation) and spanned all operable stages. The MIRO trial recapitulated many of the results of the TIME trial, reporting significantly fewer patients in the hybrid esophagectomy arm with Clavien-Dindo grade 2 (or greater) complications in the postoperative period (36\% vs 64\%). This translated to a risk-adjusted $77 \%$ lower risk of major morbidity within a 30-day period around surgery $(P<.001)$. Major pulmonary morbidity rates were also significantly lower in the hybrid arm than in the open 
esophagectomy arm (18\% vs $30 \%)$. Survival was not statistically different. It is important to note that the system of staging used in the trial was based on the American Joint Commission of Cancer edition 6, with some colloquial, unvalidated modification for involved nodes farther than $5 \mathrm{~cm}$ away from the primary being designated as $\mathrm{N} 2$. The potential disparity of lymph node number that is not accounted for in the N1 designation should not be overlooked in any aspect of future survival comparisons as this trial matures. The designation of number of lymph node number involved is an important prognostic variable that has since been introduced into the 7th and 8th editions of esophageal staging systems. ${ }^{9}$

\section{IMPLICATIONS FOR THE FUTURE OF OPEN ESOPHAGECTOMY}

Does the current literature effectively close the door on traditional open, 2-field esophagectomy in this patient population? Probably; maybe. In actuality, there was not a difference in the overall complication rate between the open and hybrid groups within the MIRO trial, a point that has been reiterated in other retrospective comparisons. ${ }^{2,3}$ There was, however, a difference in the severity of the complications when comparing the 2 cohorts. In the open group, there were more grade 2 complications, juxtaposed with more grade 1 events in the hybrid arm. The actual total numbers of events were statistically equivalent, however, and grade 3, 4, and 5 events were also equivalent. The difference in severity of postoperative event would by definition translate to more actionable complications in the open cohort. Is this a difference in the outcomes? Yes. Was it clinically significant? Maybe. Notably, the reported difference in major complications did not translate to any discrepancy in the length of stay between the 2 groups. Length of stay was equivalent. Major adverse events, such as leak, reoperation, mortality, and adult respiratory distress syndrome, were essentially the same between open and hybrid MIE cohorts. So, in fact, the outcomes from open and hybrid groups in this trial were more alike than different. There are other reasons, however, to consider converting to minimally invasive surgery. Blood loss is lower in almost every reported series and randomized trial. More importantly, quality of life is reported as better in the MIE cohort than in the open one.

\section{A PLACE FOR OPEN SURGERY?}

There were notable exclusions that are pertinent to the MIRO trial. Patients with esophagogastric junction tumors below type I were excluded, as were suspected cT4 tumors. Patients with celiac adenopathy were also excluded. In keeping an eye on surgical quality, one will note that although there is no statistical difference in complete resections, there were a few $\mathrm{R} 1$ or $\mathrm{R} 2$ resections within the hybrid
MIE group. This is despite the fact that neoadjuvant chemoradiation was often used in the hybrid arm. Chemoradiation is known to improve R0 resection rates, ${ }^{10,11}$ and it very likely facilitates the potential for an R0 hybrid resection by reducing the size of difficult, bulky lesions. The way that I interpret this data is that there are clearly areas where a surgeon's tactile feedback remains critical, and the potential lack of subtlety in the haptics that we receive from minimally invasive techniques needs to be critically assessed on a case by case and surgeon by surgeon basis. Type II lesions were avoided in this trial, perhaps because the distal gastric margin in these lesions is difficult to assess with a stapler's edge, and similarly, I have found resection of bulky celiac adenopathy difficult to navigate with sticks at times.

In full disclosure, I will say that I have transitioned much of my practice to hybrid resections. For those who know me, this may come as somewhat of a surprise. My evolution in practice was admittedly somewhat attributable to market or peer pressure. It was also, however, born out of realization that some of the technical maneuvers that seemed sacred to me and characterize a highquality open en-bloc resection could be effectively translated to minimally invasive techniques. Still others, such as drainage procedures or feeding tubes, may not be critical in every patient. As technology and systemic therapies improve, we as a body of surgeons need to continue to explore the boundaries of resectability (such as in oligometastatic disease and salvage resections) and minimize the impact of esophagectomy. During this transition, we also need to recognize that there are areas where open surgery continues to have a place-so hold off on putting the abdominal retractors into mothballs for now, and keep watching the data closely. Future trials, such as ROMIO (Randomized Oesophagectomy: Minimally Invasive or Open), will evaluate the short- and long-term outcomes of an en-bloc open esophagectomy versus a hybrid procedure, leading us to ask the question of whether minimally invasive operations and non-en bloc 2-field open operations might be an erosion away from the criterion standard. ${ }^{12}$ Above all, conversion to MIE techniques must not reduce quality of surgery and the acquisition of locoregional control of disease.

\section{CONCLUSIONS}

Our responsibility as surgeons remains maximizing value for those individual patients whom we serve. And in this surgeon's humble opinion, that requires an armamentarium of excellent surgical options from which to choose. Nonetheless, transitioning your practice to use minimally invasive techniques in appropriately selected patients should result in at least equivalent, if not improved, perioperative outcomes once the learning curve has been surmounted. 


\section{Conflict of Interest Statement}

Dr Hofstetter receives research funding and performs consulting for Ethicon and Neuwave.

\section{References}

1. Ramage L, Deguara J, Davies A, Hamouda A, Tsigritis K, Forshaw M, et al. Gastric tube necrosis following minimally invasive oesophagectomy is a learning curve issue. Ann R Coll Surg Engl. 2013;95:329-34.

2. Sihag S, Kosinski AS, Gaissert HA, Wright CD, Schipper PH. Minimally invasive versus open esophagectomy for esophageal cancer: a comparison of early surgical outcomes from the Society of Thoracic Surgeons national database. Ann Thorac Surg. 2016;101:1281-8; discussion 1288-9.

3. Seesing MFJ, Gisbertz SS, Goense L, van Hillegersberg R, Kroon HM, Lagarde SM, et al. A propensity score matched analysis of open versus minimally invasive transthoracic esophagectomy in The Netherlands. Ann Surg. 2017;266:839-46.

4. Biere SS, van Berge Henegouwen MI, Maas KW, Bonavina L, Rosman C, Garcia JR, et al. Minimally invasive versus open oesophagectomy for patients with oesophageal cancer: a multicentre, open-label, randomised controlled trial. Lancet. 2012;379:1887-92.

5. Straatman J, van der Wielen N, Cuesta MA, Daams F, Roig Garcia J, Bonavina L, et al. Minimally invasive versus open esophageal resection: three-year follow-up of the previously reported randomized controlled trial: the TIME trial. Ann Surg. 2017;266:232-6.

6. Maas KW, Cuesta MA, van Berge Henegouwen MI, Roig J, Bonavina L, Rosman C, et al. Quality of life and late complications after minimally invasive compared to open esophagectomy: results of a randomized trial. World J Surg. 2015;39:1986-93.

7. Swisher S, Ajani J, Correa A, Komaki R, Hofstetter W. Minimally invasive versus open oesophagectomy for oesophageal cancer. Lancet. 2012;380:883; author reply $885-6$.

8. Mariette C, Markar SR, Dabakuyo-Yonli TS, Meunier B, Pezet D, Collet D, et al; Fédération de Recherche en Chirurgie (FRENCH) and French Eso-Gastric Tumors (FREGAT) Working Group. Hybrid minimally invasive esophagectomy for esophageal cancer. $N$ Engl J Med. 2019;380:152-62.

9. Rice TW, Ishwaran H, Kelsen DP, Hofstetter WL, Apperson-Hansen C, Blackstone EH, Worldwide Esophageal Cancer Collaboration Investigators. Recommendations for neoadjuvant pathologic staging (ypTNM) of cancer of the esophagus and esophagogastric junction for the 8th edition AJCC/UICC staging manuals. Dis Esophagus. 2016;29:906-12.

10. Hofstetter W, Swisher SG, Correa AM, Hess K, Putnam JB Jr, Ajani JA, et al. Treatment outcomes of resected esophageal cancer. Ann Surg. 2002;236: 376-84; discussion 384-5.

11. Stahl M, Walz MK, Riera-Knorrenschild J, Stuschke M, Sandermann A, Bitzer M, et al. Preoperative chemotherapy versus chemoradiotherapy in locally advanced adenocarcinomas of the oesophagogastric junction (POET): long-term results of a controlled randomised trial. Eur J Cancer. 2017;81:183-90.

12. Avery KN, Metcalfe C, Berrisford R, Barham CP, Donovan JL, Elliott J, et al. The feasibility of a randomized controlled trial of esophagectomy for esophageal cancer-the ROMIO (randomized oesophagectomy: minimally invasive or open) study: protocol for a randomized controlled trial. Trials. 2014; $15: 200$. 